\title{
Corrigendum: Association Between Metabolic Syndrome and Mild Parkinsonian Signs Progression in the Elderly
}

\begin{abstract}
Zeyan Peng 1,2, Rui Zhou ${ }^{3}$, Dong Liu ${ }^{4}$, Min Cui ${ }^{5}, \mathrm{Ke} \mathrm{Yu}{ }^{6}$, Hai Yang ${ }^{2}$, Ling $\mathrm{Li}^{2}$, Juan Liu ${ }^{2}$, Yang Chen ${ }^{2}$, Wenjuan Hong ${ }^{1}$, Jie Huang ${ }^{1}$, Congguo Wang ${ }^{1}$, Jingjing $\mathrm{Ma}^{1}$ and Huadong Zhou ${ }^{\text {* }}$

${ }^{1}$ Department of Neurology, The First Affiliated Hospital of Bengbu Medical College, Bengbu, China, ${ }^{2}$ Department of Neurology, Army Medical Center of PLA, Chongqing, China, ${ }^{3}$ Southwest Hospital, Army Medical University, Chongqing, China, ${ }^{4}$ State Key Laboratory of Trauma, Army Medical Center of PLA, Chongqing, China, ${ }^{5}$ Department of Neurology, The General Hospital of Central Theater Command, Wuhan, China, ${ }^{6}$ Department of Neurology, The General Hospital of Western Theater Command, Chengdu, China
\end{abstract}

Keywords: metabolic syndrome, mild parkinsonian signs, Parkinson's disease, progression, elderly

\section{A Corrigendum on}

Association Between Metabolic Syndrome and Mild Parkinsonian Signs Progression in the Elderly

by Peng, Z., Zhou, R., Liu, D., Cui, M., Yu, K., Yang, H., Li, L., Liu, J., Chen, Y., Hong, W., Huang, J., Wang, C., Ma, J., and Zhou, H. (2021). Front. Aging Neurosci. 13:722836. doi: $10.3389 /$ fnagi.2021.722836

In the published article, there was an error in the affiliations of all the authors. Instead of:

Zeyan Peng ${ }^{1}$, Rui Zhou ${ }^{2}$, Dong $\mathrm{Liu}^{3}$, Min Cui ${ }^{4}$, Ke $\mathrm{Yu}^{5}$, Hai Yang ${ }^{1}$, Ling $\mathrm{Li}^{1}$, Juan Liu ${ }^{1}$, Yang Chen $^{1}$, Wenjuan Hong ${ }^{6}$, Jie Huang ${ }^{6}$, Congguo Wang ${ }^{6}$, Jingjing $\mathrm{Ma}^{6}$ and Huadong Zhou ${ }^{6} *$

${ }^{1}$ Department of Neurology, The First Affiliated Hospital of Bengbu Medical College, Bengbu, China

Frontiers Editorial Office,

Frontiers Media SA, Switzerland

*Correspondence:

Huadong Zhou

zhouhuad@163.com

Received: 06 October 2021 Accepted: 13 October 2021 Published: 24 November 2021

Citation:

Peng Z, Zhou R, Liu D, Cui M, Yu K, Yang H, Li L, Liu J, Chen Y, Hong W, Huang J, Wang $C, M a J$ and Zhou $H$

(2021) Corrigendum: Association Between Metabolic Syndrome and Mild Parkinsonian Signs Progression in the Elderly.

Front. Aging Neurosci. 13:790099.

doi: 10.3389/fnagi.2021.790099
${ }^{2}$ Department of Neurology, Army Medical Center of PLA, Chongqing, China

${ }^{3}$ Southwest Hospital, Army Medical University, Chongqing, China

${ }^{4}$ State Key Laboratory of Trauma, Army Medical Center of PLA, Chongqing, China

${ }^{5}$ Department of Neurology, The General Hospital of Central Theater Command, Wuhan, China

${ }^{6}$ Department of Neurology, The General Hospital of Western Theater Command, Chengdu, China

It should be:

Zeyan Peng ${ }^{1,2}$, Rui Zhou ${ }^{3}$, Dong $\mathrm{Liu}^{4}$, Min $\mathrm{Cui}^{5}, \mathrm{Ke} \mathrm{Yu}^{6}$, Hai Yang ${ }^{2}$, Ling $\mathrm{Li}^{2}$, Juan Liu ${ }^{2}$, Yang Chen $^{2}$, Wenjuan Hong ${ }^{1}$, Jie Huang ${ }^{1}$, Congguo Wang ${ }^{1}$, Jingjing $\mathrm{Ma}^{1}$, Huadong Zhou ${ }^{1 *}$

${ }^{1}$ Department of Neurology, The First Affiliated Hospital of Bengbu Medical College, Bengbu, China

2 Department of Neurology, Army Medical Center of PLA, Chongqing, China

${ }^{3}$ Southwest Hospital, Army Medical University, Chongqing, China

${ }^{4}$ State Key Laboratory of Trauma, Army Medical Center of PLA, Chongqing, China

${ }^{5}$ Department of Neurology, The General Hospital of Central Theater Command, Wuhan, China

${ }^{6}$ Department of Neurology, The General Hospital of Western Theater Command, Chengdu, China 
The authors apologize for this error and state that this does not change the scientific conclusions of the article in any way. The original article has been updated.

Publisher's Note: All claims expressed in this article are solely those of the authors and do not necessarily represent those of their affiliated organizations, or those of the publisher, the editors and the reviewers. Any product that may be evaluated in this article, or claim that may be made by its manufacturer, is not guaranteed or endorsed by the publisher.

Copyright $\odot 2021$ Peng, Zhou, Liu, Cui, Yu, Yang, Li, Liu, Chen, Hong, Huang, Wang, Ma and Zhou. This is an open-access article distributed under the terms of the Creative Commons Attribution License (CC BY). The use, distribution or reproduction in other forums is permitted, provided the original author(s) and the copyright owner(s) are credited and that the original publication in this journal is cited, in accordance with accepted academic practice. No use, distribution or reproduction is permitted which does not comply with these terms. 